\title{
TOWARDS THE VIRTUAL HOME: CONSTRUING THE MULTIMEDIA-HOME TO ENHANCE CULTURAL AND BIOGRAPHIC CONTINUITY
}

\author{
Kresten Bjerg \\ Institute for Psychology \\ University of Copenhagen \\ Denmark
}

\begin{abstract}
The unexpected proliferation of new electronic media in private homes under free market economies is taking place without regard for long term consumer interests.

The digital information revolution is presenting serious threats of information overload, cultural discontinuity, 'commodification' of knowledge, and colonisation of the intimate private life-worlds by impersonal and abstract systems. Further, it is mainly focused on a vertical axis of communication: information down to the consumers, money up to the commercial providers
\end{abstract}

But multimedia technologies, presently being exploited in the service of one-way mass media and commercially-dominated interactive communication, may be considered alternatively as tools available for decentralised use in private homes and local neighbourhoods. It is suggested that concepts of private homes as self-referential systems - and the construction of virtual homes and virtual neighbourhoods - may point the way to establish a grounding in real homes' and real neighbourhoods' use of technologies otherwise conducive to a disembedding of persons from psychologically important local, biographical and cultural continuity.

An experimental multimedia-home at the University of Copenhagen was in function as an early total-environment prototype for research and development along these lines until 1998.

A new Experimental Home may be created as an element in the new KUA (Copenhagen University Amager) campus currently under construction

\section{INTRODUCTION}

How can domestic informatics, telematics and automation be actively used by lay citizens to cope confidently with the chaotic complexities of the information society, in ways which are firmly grounded in their own cultural premises?

Unforeseen processes of change and innovation in information and communication technologies have consequences which necessitates an instantiated bridge-building between: 
- the philosophical, anthropological, psychological and sociological understandings of the cultural meanings and significances of the home;

- the emerging socio-political picture of a late modern information society ${ }^{1}$;

- the front-lines of technical innovations potentially available in private homes and neighbourhoods ${ }^{2}$.

\section{CHAOS AND ITS VILLAINS}

I think we have to concern ourselves with the mind-blowing anarchy, the cultural destruction and the economic and ideological seduction and exploitation which may be feared from information technology, when it effectively enters the majority of private homes.

The forces of globally free market economies, and immeasurable profits to be created by merging international consortia of privatised telecom, entertainment-industries and electronic industries, employing cheap labour in the Far East, will make this development global.

This anarchy is spreading like a forest-fire. Actually we cannot know, whether any traditional cultures will survive.

The unexpected proliferation of new electronic media in private homes under the free market economies is taking place without the faintest regard for long term consumer interests.

I have chosen the following question as my persistent and enduring research problem : how can we identify long term consumer-interests in homeoriented informatics, telematics and automation?

\section{ELECTRONIC MODERNITY}

The most basic personal growth- and life-conditions and the sustaining cultural life-forms of private households have already been deeply affected and transformed in lasting ways by the introduction of electric and electronic technologies.

For urban citizens in many western countries, the only reality we can be grounded in is one where a major part of these gadgets and their use is taken for granted and woven into a major part of our endeavours. An avalanche of increasingly complex and multipurpose information-apparatuses (photo audio-A/V- TV-VCR-computers and telephones) is now piling up in Scandinavian homes, as is also generation upon generation of storage media (photos, records, tapes, disks, hard disks). 
In the midst of this, people and families in love and care are often overloaded by attractive and imperative information through all these media, and often overwhelmed by demands and obligatory commitments to a job and a multitude of extra-familial relationships.

We have, by adopting the telephone, the radio and the TV media, and lately also computer gaming, as significant and time-demanding elements in everyday life, been brought into a peculiar placelessness, and into an exposed, one-way receiving relationship to external fiction, report and persuasion. The writings of Meyrowitz (1985), Miles (1988) Giddens (1991) and Silverstone (1992) well reflect the profound socio-psychological effects that this is having.

We assimilate and adapt through tapping numbers and letters on telephones and keyboards, flapping through pages of mass produced papers and magazines, zapping between radio and TV channels, and soon also surfing Internet; continuously reorienting and updating, finding ourselves in a global flow of commercials and contemporary as well as historical knowledge and fiction, heavily decentred outside the home.

Our senses are extended to reach from the home all around the globe and beyond. We are daily witnesses to events and narratives in an inexhaustible wealth of natural and artificial realities in the 'multiverse' of the mass media

Psychologically and sociologically all these many devices and their operation are part of a late modernity, which few of us would prefer to be without. They are used as tools for sustainment of social relations, entertainment, knowledge-acquisition, orientation, identification and the routines of general everyday survival and recreation.

\section{MULTIDIMENSIONAL VULNERABILITY AND COMPETING INTERESTS}

How can we then explore, discuss and build up an argument about long term consumer interests in these developments?

The digital information-revolution is presenting serious threats of information overload, cultural discontinuity, commodification of knowledge, and the colonisation of the intimate private life-worlds by impersonal and abstract systems.

Further, it is almost dominantly focused on a vertical axis of communication: mass and individualised information and commercials down to the consumers; personal information and money up to the commercial providers.

It is urgent to be aware of the many types of alienating and anti-socialising marginalisation which the spread of domestic technologies implies for 
already underprivileged groups of the population. All over society the general effects add to the facets of vulnerability: chaos and overload, external overdetermination, evanescence, demonisation, physical passivity, social isolation, unequal access, loss of rights, powerlessness, vulnerability to malicious or merely exploitative intent, vulnerability to suggestive marketing, dehumanisation of communication, impoverishment of social contacts, and 'Disneyfication'.

A lot of external interests will be devoted to offer, sell and exploit to each person their set of computer programs as executive interactive systems for knowledge and self-administration.

In a period of intense identity confusion they will be offering each userhousehold their special orthodox metaphysical dimensions of existence and interest as a usable compass and time-management calendar.

We can predict, that the need for 'guidance' will manifest itself in many willing victims to the producers of such 'soul-uniforming almanacs', offering their church or their party or their organisation or their school or their metaphysic as a seductive life-raft or magic flying carpet.

Each system offered and marketed will of course see its more global appropriation as a potential source for, and core of, resiliency and constructive integration.

$\mathrm{Be}$ it astrology, Tarot, fundamentalist religions and ethnocentrics, Scientology, NLP, Freemasonry or just the multitude of integrated commercial services and their fashioning and fascinating imagery which will be marketed into the home by the cables: The Integrated Service Digital Network, into which billions of ECU's are invested from Brussels, to favour economic growth and competitive advantage.

We must expect forceful economic and ideological competition in a growing market of commercial (and spiritual) offers of cheap teaching and training, support, expert advice and peddling of panaceas from the entertainment industries and the medical industries across media, as more individualised direct electronic access to and from the home is developing.

In this, the traditional role of the family home as the base of cultural continuity, anchor and embedding of growth and development of coherent personal biographic identity, is threatened.

This is the expansion of "disembedding mechanisms" which, as formulated by Giddens (1991), "prise social relations free from the hold of specific locales, recombining them across wide time-space distances... acting to transform the content and nature of day-to-day social life." .

In my view this is a development which poses serious threats to cultural continuity, tends to permeate the walls of the 'protective domestic cocoon' 
and intensifies the dangers of colonisation of the private life-world by abstract and impersonal systems.

The unavoidable intrusion of ever more powerful information and communication technologies into private homes is going to upset and transform the conditions of human childhood, adolescence, personality development and everyday life.

It will do so by creating entirely new circumstances around knowing and the knowledge acquisition of the individual, and totally new conditions for mental and social processes.

In this it may be wise to start by looking at the pictures we will be able to form concerning our selves and our own life, and concerning the relations we will be able to have with other people, other places, knowledge and cultural heritage.

The same technologies, which are presently being exploited in the service of one-way mass media and commercially dominated interactive communication, may be considered as tools available for decentralised use in private homes and local neighbourhoods.

\section{INNOVATIVE ABUNDANCIES}

It is increasingly clear that a new domestic reality awaits us around the corner.

The trends of technological innovation which are converging in the field of Home-Oriented Informatics, Telematics and Automation are linking and merging the technical developments within a wide spectrum of technologies: broadcast and point-to-point telecommunication; satellite TV with the capacity to carry 500 simultaneous channels; set-top boxes; cablechannels prepared for two-way traffic; use of the old telephone wires to carry real-time TV (ADSL).

Widespread access to mobile telephony: consumer electronics for multimodal sensory output and storage across the previously separate media-cultures ( text, graphics and animation, still- and live image, sound), connected to cheap media of individual information mass-storage in the gigabyte class

And, from another angle: environmental and physiological analogue and digital input and monitoring, controllers for feed-back regulation of sources and drains and motor-control of minor physical events in a proximal ecology. 
With the advent of video recorders, telephone answering machines, cheap video cameras, home-computers and modems, we first experience these as 'more of the same. But then comes:

- mobile telephones, lap-top notebook computers, modems, e-mail, Usenet and graphical World Wide Web 'surfing' from the home;

- an exploding market of compact disks- (CD-Roms) with interactive illustrated encyclopaedias, multilingual educational games, fascinating and involving action and adventure games, and hypermulti-media artistic productions.

- virtual reality game machines, attempting to sabotage reality-testing, and seduce young people into acting in horror-filled and murderous hero-enemy historic and fantasy-worlds.

- optical character-recognition, meaning that machines can pick up from paper not only pictures and photos, but also any typed text, recognise the letters, and speak the words.

- language translation, speech-recognition and speech-synthesis.

And, around the corner: all kinds of instructionable electronic agent-slaves in the networked computer, ready to listen to our words, obey, and report back to us, (and, uncontrollably, to others) what they found for us on today's Global Information Stock Exchange, and what they did for the consumer in Cyberspace.

The potential resultant-space, when the immense variety of primary and secondary needs emerging across the life-ages of a household are taken into account, exceeds all imagination.

\section{TURNING THE TABLES}

In this situation I find it important to create a conceptual vocabulary for thinking about the psychological, cultural and social differences that will happen and how we conceive and deal with these changes.

Looking at private homes as:

- the basic cells in the bodies of civil societies;

- the necessary basis for development and maintenance of personal identity;

- a biographically coherent frame of reference for continuous orientation in an increasingly complex wealth of information, may point a special Nordic way of seriously re-considering the attitudes to take and the strategies to choose when confronting new technological potentials in the home on a wider timescale and horizon. I propose that these new techniques shall be considered from the vantage point of how they can be actively used by lay citizens to cope confidently and on their own cultural premises with the chaotic complexities of the information society as they tend to implode in the home. 
In this we have to acknowledge and focus an imminent quantum-leap in representational power. Many of you have seen what is professionally accomplished in music videos on TV. But most of the effects demonstrated there, and a good deal more, are going to be available in multimedia homecomputers.

This applies both to various 3-D and virtual reality techniques and to so called 'hyper-linking'. We must evaluate the combination of:

- breaking out of the two-dimensionality of drawing space into threedimensional drawing and photographic montage;

- appropriating the techniques of constructing navigable annotable picture rooms;

- appropriating the 'hyperlink' technique of establishing links of reference between single pages or even single words in text and to and from places and times in picture-rooms;

- appropriating the techniques of creating and animating humanlike electronic dolls and moving them in picture-rooms.

All of this may come together in a very special way, when directed toward the representation of our own proximal life-space.

I suggest that concepts of homes as self-descriptive, self-referential systems and concepts of virtual homes and virtual neighbourhoods (as I shall explain in the following) may point the way to establish a grounding in real homes and real neighbourhoods for a technology otherwise at risk of heading us towards a devastating disembedding of persons from the most necessary local, biographical and cultural continuity.

\section{ANCHORING DOMESTIC INFORMATION ECOLOGY}

Under the threatening overload of external information-bombardment and an ever increasing rate of cultural and social change, a household is more than ever in need of a nucleus of basic orientational coordinates; a frame of reference with dimensions and directions from which to count their existential origins. The home, together with the church, has had this role but has tended to lose it. It is my hope that it can be re-instituted as a life-raft with compass and gyroscope, with the help of which a sense of purposeful navigation through life can be maintained and developed without the purpose being planted from the outside.

We can, at least in a Scandinavian-Baltic perspective think of the household and personal everyday life and socialisation in our own home as the most reliable foothold and first perspective necessary, when we look for such a 'nucleus of basic coordinates, dimensions and directions' which can be tested to be trustworthy. 
The best frame of reference for an autonomous domestic information ecology is one which is safely rooted in and modelled on the vessel of the home:

- a structured aggregate of inhabited functional rooms and spaces, with a wealth of coherently and pragmatically distributed tools and associative informative objects;

- a 'virtual mirror of the home/doll-theatre-stage' as a coherent unmarked contextualising background for any kind of expression and reflective annotation from the actor's perspective;

- a means for each household to embed its own cultural heritage and self-sedimented hyper-space of accumulated knowledge, expectations, commitments, values and valuables.

Such an orientational tool, taking the form of a home-made 3-D computer interface, may be the only resilient alternative to a multitude of 'systems for self-management' and 'mind-body multimedia work-out programs' to be offered to consumers.

\section{MERGING MEANS OF MULTIDIMENSIONAL ORIENTATION AND THE 'VIRTUAL HOME'}

We will be witnessing the merging of previously separate expressive cultures: the verbal cultures, the chireographics and paint-cultures, the newspaper, journal and book print cultures, the music cultures, the dramatic and choreographic cultures, the toy cultures (Lego, doll theatre, comic strips, animated movies). They will all converge now with, and within, the basic nuclear domestic culture.

This will gradually empower citizens to step into the role of producers of their own narrative to themselves, and to a possible future. The merging of these into one, integrated domestic multi-medium is not a question of replacing the old, separated expressive cultures, but rather that of tying them together:

- adding a superior synthesising time-perspectival level of transforming, annotating and editing, storage and retrieval potential, across the media;

- integrating the means of relocating thematic contents of actualised local relevance within a consistent local and biographical timegeography.

Under these circumstances future domestic multimedia systems should be construed and constructed with an a priori regard for their vital functions for coherent and sustainable personal orientation.

I have reached the conclusion, that this can best be accomplished through a domestic re-embedding of privately relevant information and information flows within a new kind of self-referential representational framework, a home-made multidimensional hyper-media mapping of individual homes, in 
the uniqueness of their particular interior layout, furnishing and whole cultural symbolic interior surface and storage spaces.

I suggest the notion of 'virtual homes' as designation for such frameworks, and the notion of "virtual neighbourhoods" for the possible corresponding mapping of shared proximal surroundings.

I think this can be used as a paradigm for an enculturable tool for users' autonomous existential orientation and life-space navigation from a selfbiographic home-centred ('domocentric') perspective of personally organised relevance-structures.

It should help reinstate the household members as masters of their shared and individual, inherited and acquired, information resources.

And it should enable the tying together, in a coherent conceptual pattern, of the use of the entire domestic aggregate of cultural inheritance and electronic tools.

\section{DOMESTIC SPACE RESEARCH}

The intrusion of new media in the homes will afford the home a new central role in society, a changed role in production and an entirely new role in the development of knowledge.

In this, we will increasingly meet the new and the unknown in our own and our parents' homes. We will be exposed to new orders of informationcomplexity and overload, not least in visual and audio information. And we will have practically unlimited cheap storage and retrieval capacities, which there is no established culture or tradition to deal with.

These are some of the arguments in favour of the development of new ideographic qualitative research methods, for what we might call 'domestic space research': the development and application of empirical methods to fuel the development of models and theories, descriptive and explanatory displays of the domestic life-space and its processes, and thus contribute to critical and constructive conceptualisation concerning our dealing with the psychological, social and cultural aspects of media-development for the private sphere.

My purpose is to prepare a sustainable foundation for future research and policy making in this field. 


\section{THE EXPERIMENTAL MULTIMEDIA HOME}

The thoughts presented are to a large extent built upon my experience and reflections working with an experimental habitat, 'the experimental home' at the University of Copenhagen.

This was a fully culturally settled and long time sedimented - as if inhabited - 3-room flat in the Psychological Laboratory of the University of Copenhagen, where I have been exploring the problems and potentials of information technology in the home.

The Experimental Multimedia Home constituted a tentative new species of research facility. The qualitative method and research strategies under development here were guided from an epistemological, a psychological, an ethical and an interface design perspective. And, let me add, built from an explicit humanistic multi-cultural value base.

My work in developing this laboratory facility as a 'total environment prototype' of a multimedia-home, was meant to be a pilot for later more concerted efforts directed to field experiments in flats of public housing. I am developing an electronic diary system, merging the use of text and pictograms for putting left-hemisphere and right-hemisphere reflection on a more equal footing. We were integrating computer audio and video systems with common time-codes. We were exploring ways of optimising selfreferential accountability and multimedia narrative by co-researching with voluntary student-occupants of the flat.

Contrary to what might be expected in positivistic science, occupancies were unmonitored from the outside. Our target is precisely privacy, and its free description and expression from the inhabitant-actor's perspective.

The central theme was, and is, the hyperlinkable sedimenting virtual home, as a coherent time-geographical knowledge stock interface.

I think of such private 'knowledge stock interface' as situating, embedding and rooting the household information ecology deeply and intrinsically in the soil of its native and local interior culture architecture and its generic biographical cultural and personal constructs.

But, in the longer run, I also conceive it as embedding itself, interface-wise, in its own contemporary virtual neighbourhood, and its shared, increasingly multicultural social realities. Realities which may well come to involve optimised home-link communication systems and a democratically administered sharing of common resources, territories and tools on shared inhabitant intranets.

With the recent closure of the old experimental home an era has expired. But its rationales can hardly be outdated. 


\section{OIKOS IN CYBERSPACE, PLEASE VISIT AND COLLABORATE}

I am also administering a small international researcher-network and its sedimenting platforms for knowledge-exchange and emerging taxonomy of threaded dialogues, as a part of our WWW-site. I hope that the means of orientation, which we can here collectively organise and develop, can be an investment in global long term consumer-interests in domestic infrastructure and information ecology.

At the same address I edit an electronic journal or e-zine, Oikos-e: Journal of Domestic Information Ecology, where each accepted paper will have attached a freely accessible 'threaded annotation platform'. You are cordially invited to visit our site at http//: www.psl.ku.dk/ oikos/, and to respond to its modest beginnings.

\section{NOTES}

1 which is beginning to show its early contours in the Scandinavian countries within the last few years.

2 which even to many Scandinavians sounds like pure science-fiction, but which are already more or less on the mass-production-lines in Silicon Valley and the Far East.

\section{REFERENCES}

Bjerg, K. (1996) Home-oriented informatics, telematics \& automation. In Kent, A \& Williams, J.G.(eds) Encyclopaedia of Computer Science and Technology, vol 35. Marcel Dekker, N.Y.

Giddens, A. (1991) Modernity and self-identity. Blackwell, Oxford.

Meyrowitz, J. (1985) No sense of place: the impact of electronic media on social behaviour. Oxford University Press

Miles, I. (1988) Home informatics: information technology and the transformation of everyday life. Pinter Publ., London.

Silverstone, R. et al (eds.) (1992) Consuming technologies: media and information in domestic spaces. Routledge, London. 